\title{
Commentary: CD22 blockade restores homeostatic microglial phagocytosis in ageing brains
}

\author{
Penghui Wei and Jianjun $\mathrm{Li}^{*}$ \\ Department of Anesthesiology, Qilu Hospital of Shandong University (Qingdao), Qingdao, China
}

Keywords: CD22 blockade, microglial phagocytosis, immunotherapy, neurodegenerative diseases, POCD

\section{A Commentary on}

CD22 blockade restores homeostatic microglial phagocytosis in ageing brains

\section{OPEN ACCESS}

Edited by:

Amy Lovett-Racke,

The Ohio State University,

United States

Reviewed by:

Ruth M. Barrientos,

The Ohio State University,

United States

Serge Rivest,

Laval University, Canada

*Correspondence:

Jianjun Li

lij9573@163.com

Specialty section

This article was submitted to

Multiple Sclerosis and

Neuroimmunology,

a section of the journal

Frontiers in Immunology

Received: 24 April 2019

Accepted: 22 May 2019

Published: 06 June 2019

Citation:

Wei P and Li J (2019) Commentary: CD22 blockade restores homeostatic

microglial phagocytosis in ageing brains. Front. Immunol. 10:1301

doi: 10.3389/fimmu.2019.01301 by Pluvinage, J. V., Haney, M. S., Smith, B. A. H., Sun, J., Iram, T., Bonanno, L., et al. Nature (2019). 568, 187-192. doi: 10.1038/s41586-019-1088-4

Recently, a ground-breaking study on microglia being a potential target for immunotherapy in neurodegenerative diseases was reported in Nature (1). Pluvinage et al. combined CRISPR-Cas9 knockout screens with RNA sequencing analysis to identify approximately 3,000 genes. They finally discovered that $\mathrm{CD} 22$, a canonical receptor typically expressed on B-cells (2), was significantly upregulated in microglia of the central nervous system (CNS) of an aged mouse, and approximately three times more in aged microglia than young microglia. Interestingly, the authors showed that $\mathrm{CD} 22$ was a negative regulator of microglial phagocytosis, and blocking CD22 in CNS either by specific antibody or by genetic ablation promoted the clearance of extracellular oligomeric amyloid- $\beta$ [A $\beta$, neuropathological hallmark of Alzheimer's disease (AD)] and $\alpha$-synuclein fibrils (pathological hallmark of Parkinson's disease) in vivo, and improved hippocampal-dependent learning and memory performance. Furthermore, they found that the mechanism underlying microglial CD22-blocking-mediated neuroprotection may involve an increase in the number of hippocampal dentate granule neurons expressing c-FOS and phospho-cAMP response element-binding protein. The research represents a breakthrough in the field of treatment for neurodegenerative diseases. The immunotherapy conducted by microglial CD22 may be a potential therapeutic strategy to restore and improve age-related cognitive impairment.

Perioperative neurocognitive disorder, an age-related neurodegenerative disease, is a highly prevalent condition with significant effects on the prognosis of elderly individuals undergoing anesthesia and surgery, including acute post-operative delirium and long-lasting post-operative cognitive dysfunction (POCD) $(3,4)$. The incidence of POCD was reportedly between 25 and $40 \%$ among elderly patients at the point of discharge, and it could lead to a significantly higher mortality rate (5). The exact mechanism of POCD remains elusive and there is no agreement on the efficiency of current treatments such as anti-inflammatory, antioxidant and neuroprotective agents (6). $A \beta$ and phosphorylation of tau protein, secondary to anesthesia and surgery, are believed to be pathological hallmarks of POCD (7). Normal microglia engulf and degrade $A \beta$ and tau protein by 
phagocytosis; however, the change in engulfment of microglia is associated with age and the aged microglia become more proinflammatory and less phagocytic $(1,8)$. Therefore, we speculated that the age-related deterioration of microglial phagocytosis could be the potential mechanism underlying POCD. Immunotherapy mediated by the restoration of microglial phagocytosis could provide a new path in preventing and treating POCD.

Immunotherapy induced by selective blockade of some immune checkpoints, such as PD-1/PD-L1, is commonly utilized to mobilize the immune system against a variety of malignancies (9). Research examining the use of immunotherapy in neurodegenerative diseases, such as $\mathrm{AD}$ and POCD, seems promising but appears relatively under-developed as a field. A ground-breaking report in Nature Medicine in 2015 showed that the PD-1 pathway blockade evoked an interferon- $\gamma$-associated systemic immune response, induced CNS recruitment of myeloid cell, and restrained accumulation of cerebral $A \beta$, which contributed to improvement of cognitive performance in heterozygous 5XFAD transgenic mouse models of $\mathrm{AD}$ (10). A recent study has also demonstrated that immune checkpoint blockade targeting the $\mathrm{PD}-1 / \mathrm{PD}-\mathrm{L} 1$ pathway resulted in increased immunomodulatory monocyte-derived macrophages within the brain parenchyma and combated cognitive impairment in mouse models of both $\mathrm{AD}$ and tauopathy (11). However, the available evidence is inconsistent. Gomez-Nicol et al. observed no apparent impact of PD1 deficiency on murine prion disease (ME7 strain). On the contrary, they found a slight exacerbation of the cognitive performance of ME7 mice upon PD-1 deficiency (12). The conflicting findings may attribute to different animal models presenting neurodegenerative diseases. Another potential reason could be non-specific expression of PD-1 on immune cells in the aged mouse, which is widely expressed in dendritic cells, B-cells, activated T-cells (both CD4+ and CD8+), and natural killer cells (9). The CD22 blockade appears to be more promising

\section{REFERENCES}

1. Pluvinage JV, Haney MS, Smith BAH, Sun J, Iram T, Bonanno L, et al. CD22 blockade restores homeostatic microglial phagocytosis in ageing brains. Nature. (2019) 568:187-92. doi: 10.1038/s41586-019-1088-4

2. Macauley MS, Crocker PR, Paulson JC. Siglec-mediated regulation of immune cell function in disease. Nat Rev Immunol. (2014) 14:653-66. doi: $10.1038 /$ nri3737

3. Evered L, Silbert B, Knopman DS, Scott DA, DeKosky ST, Rasmussen LS, et al. Recommendations for the nomenclature of cognitive change associated with anaesthesia and surgery-2018. Anesthesiology. (2018) 129:8729. doi: 10.1097/ALN.0000000000002334

4. Xiang X, Yu Y, Tang X, Chen M, Zheng Y, Zhu S. Transcriptome profile in hippocampus during acute inflammatory response to surgery: toward early stage of PND. Front Immunol. (2019) 10:149. doi: 10.3389/fimmu.2019.00149

5. Skvarc DR, Berk M, Byrne LK, Dean OM, Dodd S, Lewis M, et al. Post-operative cognitive dysfunction: an exploration of the inflammatory hypothesis and novel therapies. Neurosci Biobehav Rev. (2018) 84:116-33. doi: 10.1016/j.neubiorev.2017.11.011

6. Wei P, Yang F, Zheng Q, Tang W, Li J. The potential role of the NLRP3 inflammasome activation as a link between mitochondria ROS generation than PD-1/PD-L1 blockade, and may exhibit better efficiency and lesser side effects as a therapeutic strategy in neurodegenerative diseases. Although CD22 is typically expressed in B-cells, it is specifically upregulated in aged microglia (1). Pluvinage et al. observed that blocking CD22 specifically in the CNS via cerebral injection, rather than blocking CD22 systemically via intraperitoneal injection, contributed to cognitive improvement in aged mice that showed that peripheral B-cells did not mediate neuroprotective effect of anti-CD22 treatment (1). Moreover, pro-phagocytic effect of anti-CD22 treatment was mainly mediated by resident microglia and not the infiltrating peripheral macrophages (13). Furthermore, the upregulated CD22 expression in microglia depends on age. The study showed that aged microglia increased the number of CD22 surface molecules approximately three times more than young microglia, and CD22 blockade treatment had effects that were more significant in the CNS of the aged mouse than that of the younger mouse (1). Above all, aged microglia specifically expressing CD22 in CNS could be a potential and viable molecular target in neurodegenerative diseases, especially for $\mathrm{AD}$ and POCD. However, the effect of anti-CD22 treatment on age-related diseases needs to further studies involving different animal models.

\section{AUTHOR CONTRIBUTIONS}

PW drafted the manuscript. JL revised the manuscript. All authors read and approved the final manuscript.

\section{FUNDING}

This work was supported by grants from People's Benefit Project of Science and Technology in Qingdao (18-6-1-74-nsh) and the Scientific Research Foundation of Qilu Hospital of Shandong University (Qingdao) (Grant Nos. QDKY2016QN01 and QDKY2016ZD05). and neuroinflammation in postoperative cognitive dysfunction. Front Cell Neurosci. (2019) 13:73. doi: 10.3389/fncel.2019.00073

7. Xie Z, McAuliffe S, Swain CA, Ward SA, Crosby CA, Zheng H, et al. Cerebrospinal fluid abeta to tau ratio and postoperative cognitive change. Ann Surg. (2013) 258:364-9. doi: 10.1097/SLA.0b013e31 $8298 \mathrm{~b} 077$

8. Marsh SE, Abud EM, Lakatos A, Karimzadeh A, Yeung ST, Davtyan H, et al. The adaptive immune system restrains Alzheimer's disease pathogenesis by modulating microglial function. Proc Natl Acad Sci USA. (2016) 113:E131625. doi: 10.1073/pnas.1525466113

9. Constantinidou A, Alifieris C, Trafalis DT. Targeting programmed cell death-1 (PD-1) and ligand (PD-L1): a new era in cancer active immunotherapy. Pharmacol Ther. (2019) 194:84-106. doi: 10.1016/j.pharmthera.2018.09.008

10. Baruch K, Deczkowska A, Rosenzweig N, Tsitsou-Kampeli A, Sharif AM, Matcovitch-Natan O, et al. PD-1 immune checkpoint blockade reduces pathology and improves memory in mouse models of Alzheimer's disease. Nat Med. (2016) 22:135-7. doi: 10.1038/nm.4022

11. Rosenzweig N, Dvir-Szternfeld R, Tsitsou-Kampeli A, Keren-Shaul $\mathrm{H}$, Ben-Yehuda $\mathrm{H}$, Weill-Raynal $\mathrm{P}$, et al. PD-1/PD-L1 checkpoint blockade harnesses monocyte-derived macrophages to combat cognitive 
impairment in a tauopathy mouse model. Nat Commun. (2019) 10:465. doi: 10.1038/s41467-019-08352-5

12. Obst J, Mancuso R, Simon E, Gomez-Nicola D. PD-1 deficiency is not sufficient to induce myeloid mobilization to the brain or alter the inflammatory profile during chronic neurodegeneration. Brain Behav Immun. (2018) 73:708-16. doi: 10.1016/j.bbi.2018.08.006

13. Bennett ML, Bennett FC, Liddelow SA, Ajami B, Zamanian JL, Fernhoff $\mathrm{NB}$, et al. New tools for studying microglia in the mouse and human CNS. Proc Natl Acad Sci USA. (2016) 113:E1738-46. doi: 10.1073/pnas.152 5528113
Conflict of Interest Statement: The authors declare that the research was conducted in the absence of any commercial or financial relationships that could be construed as a potential conflict of interest.

Copyright (c) 2019 Wei and Li. This is an open-access article distributed under the terms of the Creative Commons Attribution License (CC BY). The use, distribution or reproduction in other forums is permitted, provided the original author $(s)$ and the copyright owner(s) are credited and that the original publication in this journal is cited, in accordance with accepted academic practice. No use, distribution or reproduction is permitted which does not comply with these terms. 